\title{
SUBSIDY IN LICENSING: OPTIMALITY AND WELFARE IMPLICATIONS*
}

\author{
by \\ CHUN-HSIUNG LIAO \\ National Cheng Kung University \\ and \\ DEBAPRIYA SEN $N^{\dagger}$ \\ State University of New York at Stony Brook
}

\begin{abstract}
This paper shows that subsidy can naturally emerge as part of the equilibrium strategy of an innovator of a cost-reducing innovation in a Cournot oligopoly when the innovator is endowed with combinations of upfront fee and royalty. It is further shown that there are robust regions where the social welfare is higher in subsidy-based licensing compared to the regime where licensing involving subsidy is not allowed. The analysis is carried out for both outsider and incumbent innovators.
\end{abstract}

\section{Introduction}

Subsidy plays an important role in R\&D. There is a large, and growing, literature that investigates the effectiveness of R\&D subsidy as a welfareenhancing instrument in oligopolies, especially in the context of strategic trade (e.g. Spencer and Brander, 1983; Bagwell and Staiger, 1992; Leahy and Neary, 1997; Muniagurria and Singh, 1997; Mukherjee, 2002). ${ }^{1}$ A subsidy is usually considered to be a policy available to the government and as such it necessarily entails intervention. The aim of this paper is to bring out a phenomenon that is in contrast with the generally perceived notion of a subsidy: we show that in a specific context of $R \& D$, namely, licensing of a costreducing technological innovation, a subsidy can naturally emerge as part of the equilibrium strategy of the innovator without any outside intervention. Moreover, it is shown that there are robust regions where the social welfare with subsidy is higher compared to the regime where licensing involving subsidy is not allowed. We carry out our analysis in a Cournot oligopoly framework under two scenarios: the innovator is an outsider to the industry and the case where she is one of the incumbent producers. Specifically, we consider a Cournot oligopoly where there are two firms other than the inno-

\footnotetext{
* Manuscript received 26.6.03; final version received 15.10.04.

We wish to thank Yair Tauman and an anonymous referee for several insightful comments and suggestions. The first author gratefully acknowledges partial financial support from the National Science Council (Grant NSC 91-2415-H-006-001) and the MOE Program for Promoting Academic Excellence of Universities (Grant 91-H-FA08-1-4).

${ }^{1}$ This list is only meant to be indicative and by no means exhaustive.
} 
vator. ${ }^{2}$ Initially, all firms produce with an identical constant marginal cost. The innovator has a non-drastic cost-reducing innovation ${ }^{3}$ that she intends to sell to some or all other firms in the industry. The licensing schemes available to the innovator are the set of all linear two-part tariffs, i.e. combinations of an upfront fee and a per-unit uniform linear royalty. It is shown that in a Cournot duopoly with an outsider innovator, the optimal licensing policy involves selling the license to only one firm using a negative per-unit royalty; in other words, the innovator pays the sole licensee a subsidy for every unit that it produces. The same is true in the case of an incumbent innovator with two other firms when the innovation is not too significant. Comparing the social welfare with the scenario when licensing involving subsidy is not allowed, we show that in the case of an outsider innovator, the restriction on subsidy leads to a reduction in welfare when the innovation is not very significant, while it improves welfare for relatively significant innovations. In the case of an incumbent innovator, the result is sharper: the restriction on subsidy is never welfare-improving. ${ }^{4}$

The formal analysis of patent licensing was initiated by Arrow (1962), who argued that a perfectly competitive industry provides a higher incentive to innovate than a monopoly. The theoretical literature of licensing has mainly considered three standard modes of licensing: royalty (Kamien and Tauman, 1984, 1986), upfront fee and auction (Katz and Shapiro, 1985, 1986). It has been shown that for an outsider innovator royalty licensing is inferior to both auction and upfront fee (Kamien and Tauman, 1984, 1986; Kamien et al., 1992). ${ }^{5}$ On the other hand, royalty licensing could yield higher payoff when the innovator is one of the incumbent firms in the industry (Shapiro, 1985; Wang, 1998; Kamien and Tauman, 2002; Sen, 2002). In a companion paper (Sen and Tauman, 2003), optimal combinations of upfront fee and royalty have been considered for both outsider and incumbent innovators, but the rate of royalty has been restricted to take only non-negative values - an assumption that has been always implicitly maintained in the existing literature of licensing. The present paper shows that endowing the innovator with a broader set of licensing policies where negative royalties are

\footnotetext{
${ }^{2}$ At least two firms are needed to capture the effect of competition for the new technology. We consider only two firms for clarity of presentation and our main results will continue to hold qualitatively for larger oligopolies.

${ }^{3} \mathrm{~A}$ cost-reducing innovation is said to be drastic (Arrow, 1962) if the monopoly price under the new technology does not exceed the competitive price under the old technology; otherwise, it is non-drastic. Clearly, if an incumbent innovator is endowed with a drastic costreducing innovation, she earns the monopoly profit with the new technology. The same fact is true for an outsider innovator when the industry size is at least two. So, the analysis of licensing is non-trivial only when the innovation is non-drastic.

${ }^{4}$ It can be shown that when the industry size is sufficiently large (in particular, at least seven), for both outsider and incumbent innovators, subsidy-based licensing always results in higher welfare.

${ }^{5}$ See, however, the recent paper of Sen (forthcoming), where it is argued that royalty could be superior to both auction and fee for an outsider innovator.
} 
allowed leads to an improvement in the payoff of the innovator as well as the overall social welfare.

While we investigate the effect of subsidy in the specific context of patent licensing, in spirit, our analysis is closely related to the principal-agent literature (e.g. Wilson, 1968; Ross, 1973; Mirrlees, 1974; Hart and Holmstrom, 1987). The interaction of the innovator with the firms can be viewed as a principal-agent model where the principal (innovator) can sign a contract with the agents (firms) through a licensing policy, but the agents cannot contract among themselves. As we discuss later in more detail, by offering a perunit subsidy together with the innovation, the innovator makes a licensee more efficient and that increases the willingness to pay for the license - this is similar to the effect caused by a principal who manipulates the marginal payoff of an agent to make him more aggressive (Fershtman and Judd, 1987).

The rest of the paper is organized as follows. We describe the model in Section 2. Optimal licensing schemes are described in Section 3. Welfare implications of subsidy-based licensing are discussed in Section 4. All proofs have been relegated to the Appendix.

\section{The Model}

We describe the model with an outsider innovator in detail. The model with an incumbent innovator will be similar, except for some obvious modifications. We consider a Cournot duopoly where $\{1,2\}$ is the set of firms and both firms produce the same product. For $i \in\{1,2\}$, let $q_{i}$ be the quantity produced by firm $i$ and let $Q=q_{1}+q_{2}$. The demand function of the industry is given by $Q=a-p$, for $p \leq a$, and $Q=0$ otherwise. With the old technology, both firms produce with the identical constant marginal cost $c$, where 0 $<c<a$. An outsider innovator has a patent for a new technology that reduces the marginal cost from $c$ to $c-\varepsilon$, where $0<\varepsilon<c$. The innovation is assumed to be non-drastic, i.e. the monopoly price under the new technology $(a+c-$ e) $/ 2$ exceeds the competitive price under the old technology $c$, implying that $a-c>\varepsilon$. The innovator decides to license the new technology to one or both firms of the industry. In the case of an incumbent innovator, we have three firms, where $\{I, 1,2\}$ is the set of firms and firm $I$ is the innovator.

\subsection{The Licensing Schemes}

The set of licensing schemes available to the innovator is the set of all linear two-part tariffs, i.e. combinations of an upfront fee and a per-unit uniform linear royalty. The schemes can be classified into two types on the basis of how the upfront fee is determined: (i) the upfront fee plus royalty (FR) policy and (ii) the auction plus royalty (AR) policy.

A typical FR policy is given by $\langle m, r, f\rangle$, where $m(=1,2)$ is the number of firms to whom the policy is offered, $r$ is the per-unit uniform royalty and 
$f$ is the upfront fee that each licensee has to pay. For this policy, the innovator first chooses a subset of $m(=1,2)$ firm(s) and makes a binding commitment that she will not sell the license to any firm outside the chosen subset. Then she offers each firm in this subset the policy $\langle m, r, f\rangle$, which the firms accept or reject simultaneously. If a licensee produces $q$, it pays the innovator $f+r q$.

A typical AR policy is given by $\langle m, r\rangle$, where $m(=1,2)$ is the number of firms to whom the policy is offered and $r$ is the per-unit uniform royalty that each licensee has to pay. When the policy $\langle m, r\rangle$ is announced, firms bid for the license in a first-price sealed-bid auction and $m$ highest bidders win the license (ties are resolved at random). If a firm wins the license with bid $b$ and produces $q$, it pays the innovator $b+r q$.

Observe that when the rate of royalty $r$ is negative, a licensee receives $-r$ from the innovator for every unit that it produces. We can then formally define subsidy as follows.

Definition: We say that a licensing policy involves a per-unit subsidy if the rate of royalty $r$ is negative and $s=-r$ is called the rate of subsidy.

To investigate the optimality and welfare implications of a subsidy in licensing, we consider two regimes: 1 and 2. Under Regime 1, the innovator can charge any $r \in \Re$ as royalty. In particular, under this regime, the innovator is allowed to provide a per-unit subsidy to a potential licensee by charging $r<0$. Under Regime 2, the innovator can only charge $r \geq 0$ as royalty, so that subsidies are not allowed under this regime.

\subsection{The Willingness to Pay for the License}

Now we determine the willingness to pay for the license. Observe that the Cournot output and profit of any firm depend on the number of licensees $m$ and the rate of royalty $r$, but they are independent of the upfront fee or the winning bid. Let us denote by $\Phi_{\mathrm{L}}(m, r)$ and $\Phi_{\mathrm{N}}(m, r)$ the Cournot profit of a licensee and a non-licensee respectively when the number of licensees is $m$ and the rate of royalty is $r$. Let $q_{\mathrm{L}}(m, r)$ and $q_{\mathrm{N}}(m, r)$ denote the corresponding Cournot outputs. ${ }^{6}$

First consider the case where the innovator seeks to sell only one license (i.e. $m=1$ ) and suppose the rate of royalty is $r$. To execute this through the FR policy, the innovator chooses one firm and makes a binding commitment that she will not sell the license to the other firm. This implies that if the chosen firm rejects the offer, no firm has a license, in which case the Cournot profit of any firm is $\Phi_{\mathrm{N}}(0, r)$. Since the Cournot profit of a firm when it is

${ }^{6}$ Of course, these expressions will be different depending on whether the innovator is an incumbent firm or not. We use the same notation to avoid notational complication.

(C) Blackwell Publishing Ltd and The University of Manchester, 2005. 
the sole licensee is $\Phi_{\mathrm{L}}(1, r)$, it follows that for the FR policy the willingness to pay for the license is

$$
f(1, r)=\Phi_{\mathrm{L}}(1, r)-\Phi_{\mathrm{N}}(0, r)
$$

So for $m=1$ and rate of royalty $r$, the optimal FR policy for the innovator is $\langle 1, r, f(1, r)\rangle$. Next observe that in case of the AR policy the willingness to pay for the license is

$$
b(1, r)=\Phi_{\mathrm{L}}(1, r)-\Phi_{\mathrm{N}}(1, r)
$$

In contrast to (1), here we subtract $\Phi_{\mathrm{N}}(1, r)$ instead of $\Phi_{\mathrm{N}}(0, r)$, because for the AR policy $\langle 1, r\rangle$ a firm knows that, irrespective of whether it becomes a licensee or not, there will always be one licensee. When the AR policy $\langle 1, r\rangle$ is announced, in equilibrium, both firms bid $b(1, r)$ and one of them is chosen at random to be the sole licensee. ${ }^{7}$ From our discussion so far, it follows that the difference in payoffs from AR and FR policies is $b(1, r)-f(1, r)$. Comparing (1) and (2), we have

$$
b(1, r)-f(1, r)=\Phi_{\mathrm{N}}(0, r)-\Phi_{\mathrm{N}}(1, r)
$$

Note that the effective marginal cost of a licensee is $c-\varepsilon+r$ and that of a non-licensee is $c$. As long as $r \leq \varepsilon$, a licensee is at least as efficient as a nonlicensee. Hence the Cournot profit of a non-licensee is decreasing in the number of licensees, so that $\Phi_{\mathrm{N}}(0, r) \geq \Phi_{\mathrm{N}}(1, r)$, which in turn implies that $b(1, r) \geq f(1, r)$. The following lemma, whose proof appears in the Appendix, follows from the fact that the optimal level of royalty does not exceed the magnitude of the innovation $\varepsilon$.

Lemma 1: Let $\Pi_{\mathrm{FR}}(1, r)$ and $\Pi_{\mathrm{AR}}(1, r)$ denote the payoff of the innovator from the FR and AR policy respectively when there is only one licensee and the rate of royalty is $r$. Then,

$$
\max _{r \in \Re} \Pi_{\mathrm{FR}}(1, r) \leq \max _{r \in \Re} \Pi_{\mathrm{AR}}(1, r) \quad \text { and } \quad \max _{r \geq 0} \Pi_{\mathrm{FR}}(1, r) \leq \max _{r \geq 0} \Pi_{\mathrm{AR}}(1, r)
$$

We then conclude that if the innovator sells the license to only one firm, the AR policy yields higher payoff than the FR policy under both regimes. ${ }^{8}$ This conclusion holds for both outsider and incumbent innovators.

${ }^{7}$ If only one firm bids, it will win the license with any bid, so it will bid zero. But then the other firm can win by bidding any small positive amount. Thus, in equilibrium, both firms must bid. Now if the second highest bid is smaller than the highest bid, then the highest bidder can benefit from a small reduction of his bid, which shows that in equilibrium both firms will bid the same amount and this amount must equal the willingness to pay.

${ }^{8}$ This result is well known in the licensing literature and actually holds more generally: in an $n$ firm oligopoly, whenever the number of licensees is at most $n-1$, the AR policy yields higher payoff than the FR policy. Katz and Shapiro (1986) showed the superiority of auction over fixed fee without any royalty and the same intuition can be carried over when the licensing policies involve royalty. 
Now consider the case where the innovator seeks to sell the license to both firms (i.e. $m=2$ ). Using a similar argument as before, we conclude that for the rate of royalty $r$ the optimal FR policy is given by $\langle 2, r, f(2, r)\rangle$, where

$$
f(2, r)=\Phi_{\mathrm{L}}(2, r)-\Phi_{\mathrm{N}}(1, r)
$$

If the innovator announces she will auction off two licenses, no firm will place a positive bid since each one is guaranteed to have a license irrespective of its bid. So the innovator can use the AR policy for $m=2$ only with a prespecified minimum bid, say $\underline{b}$. Let $\langle 2, r, \underline{b}\rangle$ be the modified AR policy with the minimum bid. When this policy is announced, in equilibrium, every firm bids exactly $\underline{b}$. The optimal value of $\underline{b}$ is $f(2, r)$, as this is the maximum fee that a firm will pay when the license is sold to both firms. We then conclude that for $m=2$, FR and AR policies coincide.

To sum up, it is thus sufficient to consider the AR policy $\langle 1, r\rangle$ for $m=$ 1 and the modified AR policy $\langle 2, r, f(2, r)\rangle$ for $m=2$, where $f(2, r)$ is given by (4). Then the payoff of an outsider innovator when she sells only one license with the rate of royalty $r$ is

$$
\Pi_{\mathrm{O}}(1, r)=r q_{\mathrm{L}}(1, r)+\Phi_{\mathrm{L}}(1, r)-\Phi_{\mathrm{N}}(1, r)
$$

while her payoff when she sells the license to both firms is

$$
\Pi_{\mathrm{O}}(2, r)=2 r q_{\mathrm{L}}(2, r)+2\left[\Phi_{\mathrm{L}}(2, r)-\Phi_{\mathrm{N}}(1, r)\right]
$$

Let $\Phi_{\mathrm{I}}(m, r)$ be the Cournot profit of an incumbent innovator when the number of licensees is $m$ and the rate of royalty is $r$. Then the corresponding payoffs for an incumbent innovator are given by

$$
\begin{aligned}
& \Pi_{\mathrm{I}}(1, r)=\Phi_{\mathrm{I}}(1, r)+r q_{\mathrm{L}}(1, r)+\Phi_{\mathrm{L}}(1, r)-\Phi_{\mathrm{N}}(1, r) \\
& \Pi_{\mathrm{I}}(2, r)=\Phi_{\mathrm{I}}(2, r)+2 r q_{\mathrm{L}}(2, r)+2\left[\Phi_{\mathrm{L}}(2, r)-\Phi_{\mathrm{N}}(1, r)\right]
\end{aligned}
$$

\subsection{The Games $G^{\mathrm{O}}$ and $G^{\mathrm{I}}$}

Now we describe the licensing games for outsider and incumbent innovators. The licensing game with an outsider innovator, denoted by $G^{\mathrm{O}}$, has three stages. In the first stage, the innovator announces either $\langle 1, r\rangle$ or $\langle 2, r, f(r)\rangle$, where $r \in \mathfrak{R}$ under Regime 1 and $r \geq 0$ under Regime 2. In the second stage, firms in $\{1,2\}$ bid simultaneously for the license, where the $m$ highest bidders win the license and pay their respective bids $(m=1$ or 2 depending on the policy). Ties in the cutoff bid are resolved at random. The set of licensees becomes commonly known at the end of the second stage. In the third stage, all firms compete in quantities. If a licensee produces $q$, it pays $r q$ to the innovator apart from its winning bid. The licensing game $G^{\mathrm{I}}$ with an incumbent innovator is defined similarly. Note that for the game $G^{\mathrm{I}}$, in the third stage, the innovator produces with marginal cost $c-\varepsilon$ and competes with all other 
firms. For both games $G^{\mathrm{O}}$ and $G^{\mathrm{I}}$, we employ the backward induction method to find the subgame-perfect equilibrium outcomes.

\section{Optimal Licensing Schemes}

\section{An Outsider Innovator}

Proposition 1: Suppose $a-c>\varepsilon$. The game $G^{\mathrm{O}}$ has a unique subgame-perfect equilibrium outcome. In this outcome, the following hold, where $r_{O}^{*}$ is the equilibrium rate of royalty.

1.) Under Regime 1, the innovator sells the license to only one firm and $r_{O}^{*}<0$. When $\varepsilon<(a-c) / 2, r_{O}^{*}=-(a-c) / 2$ and both firms continue to operate. When $\varepsilon \geq(a-c) / 2, r_{O}^{*}=\varepsilon-(a-c)$, the Cournot price is $c$ and the non-licensee firm drops out of the market.

2.) Under Regime 2, the innovator sells the license to both firms and they continue to operate. When $\varepsilon \leq(a-c) / 3, r_{\mathrm{O}}^{*}=0$, and when $\varepsilon>(a-c) / 3$, $r_{\mathrm{O}}^{*}=\varepsilon / 2-(a-c) / 6>0$.

\section{An Incumbent Innovator}

Proposition 2: Suppose $a-c>\varepsilon$. The game $G^{\mathrm{I}}$ has a unique subgame-perfect equilibrium outcome. In this outcome, the following hold, where $r_{1}^{*}$ is the equilibrium rate of royalty.

1. There is $k>2$ such that, under Regime 1 , the innovator sells the license to only one firm and $r_{1}^{*}=[2 \varepsilon-(a-c)] / 3<0$ when $\varepsilon<(a-c) / k$. When $\varepsilon$ $\geq(a-c) / k$, the innovator sells the license to both firms using the royalty policy with $r_{1}^{*}=\varepsilon$. In both cases, all firms continue to operate.

2. Under Regime 2, the innovator sells the license to both firms using the royalty policy with $r_{1}^{*}=\varepsilon$ and all firms continue to operate.

Let us now provide some intuition behind Propositions 1 and 2. Since the firms are competing in quantities in a Cournot oligopoly under linear demand, every firm regards the product of any competitor as a strategic substitute (Bulow et al., 1985). That is, a higher output from competitors lowers the marginal profit of a firm and as a consequence the best response function of any firm is downward sloping in the output of other firms. A reduction in the cost of a firm shifts its best response function to the right and results in higher Cournot output for itself and lower output for its competitors. Due to these effects, the marginal profit of the firm goes up. Observe that the rate of royalty, by entering the marginal cost of a licensee, determines its effective cost. When the innovator provides a per-unit subsidy to a firm together with the innovation, its effective cost is reduced even more. This 
leads to a further rightward shift of the best response function and a consequent increase in the marginal profit. In spirit, this is similar to the effect when a principal manipulates the marginal payoff of an agent to make him more aggressive (as in Fershtman and Judd, 1987). ${ }^{9}$ The use of subsidy can be effective in this way, because the innovator has another instrument in the form of an upfront fee (which could be the winning bid of an auction). A subsidy results in a loss from royalty payments on the one hand, but on the other it makes a potential licensee more efficient, which in turn increases the willingness to pay for the license and results in a higher upfront fee. The latter effect is dominant especially when the innovation is relatively insignificant, because then the innovation itself does not provide a licensee with sufficient competitive edge over its rivals.

\section{Welfare Comparison of the Regimes}

Now we are in a position to discuss the welfare implications of subsidy-based licensing. Let us first formally define social welfare.

Definition: Social welfare is the sum of the innovator's payoff, other firms' payoffs and consumer surplus.

Proposition 3: Suppose $a-c>\varepsilon$. For $i=1,2$, let $W_{i}$ denote the social welfare under Regime $i$. Then the following hold.

1. For both games $G^{\mathrm{O}}$ and $G^{\mathrm{I}}$, the innovator is always better off under Regime 1 and the firms are always better off under Regime 2 .

2. For the game $G^{\mathrm{O}}$, the consumers are better off under Regime 1 if $\varepsilon \leq$ $2(a-c) / 3$ and worse off under this regime if $\varepsilon>2(a-c) / 3$. For the game $G^{\mathrm{l}}$, the consumers are always better off under Regime 1 .

3. For the game $G^{\mathrm{O}}, W_{1} \geq W_{2}$ for $\varepsilon \leq 2(a-c) / 3$ and $W_{1}<W_{2}$ for $\varepsilon>$ $2(a-c) / 3$, with equality iff $\varepsilon=2(a-c) / 3$.

4. For the game $G^{\mathrm{I}}$, there is $k>2$ such that $W_{1}>W_{2}$ for $\varepsilon<(a-c) / k$ and $W_{1}=W_{2}$ for $\varepsilon \geq(a-c) / k$.

That the innovator is better off under Regime 1 is obvious, since this regime provides her with a larger set of licensing policies. Observe from (2) and (4) that when the equilibrium rate of royalty is $r$, regardless of whether the number of licensees is one or two, the net payoff of any firm is $\Phi_{\mathrm{N}}(1, r)$, which is the Cournot profit of a non-licensee when there is only one licensee. The effective cost of a licensee is $c-\varepsilon+r$, implying that $\Phi_{\mathrm{N}}(1, r)$ is increasing in $r$. Since the equilibrium rate of royalty is higher under Regime 2, it follows that firms are better off under this regime. Let us now consider the

${ }^{9}$ We are grateful to an anonymous referee for this intuition.

(C) Blackwell Publishing Ltd and The University of Manchester, 2005. 
consumers. The cost of the innovator, when the innovator is an incumbent producer, stays the same under both regimes. Under Regime 1, a licensee is very efficient due to subsidy, while a non-licensee operates under the old cost. On the other hand, under Regime 2, both firms are licensees, but due to a non-negative rate of royalty, both are moderately efficient. Thus, unlike the cases of the innovator or the firms, the effect of different regimes on consumers is not immediate, at least for the small sizes of industry that we are considering. In particular, observe that for relatively significant innovations, in the case of an outsider innovator, subsidization results in a monopoly where the price equals the pre-innovation cost $c$. This is the maximum price that leads the non-licensee to drop out of the market and it does not pay the innovator to subsidize the licensee further to bring down the price below $c$. Under Regime 2, however, both firms are licensees, and for sufficiently significant innovations the duopoly price can fall below $c$ leading to a higher consumer surplus, as stated in part 2 of Proposition 3 . This is precisely the reason why the social welfare might be higher in Regime 2 . To see this more clearly, let us now compare the welfare of two regimes. Let $p(m, r), Q(m, r)$ and $W(m, r)$ denote the Cournot price, industry output and social welfare respectively when there are $m$ licensees and the rate of royalty is $r .{ }^{10}$ Since an upfront fee or a winning bid from an auction is a lump-sum transfer from one agent to another, we can ignore them for computing the welfare. Noting that the consumer surplus is given by $Q(m, r)^{2} / 2$ and using the notations introduced in Section 2, we have the following (where $\Phi_{1}(m, r)=0$ when the innovator is an outsider).

$$
\begin{aligned}
W(m, r)= & \Phi_{\mathrm{I}}(m, r)+m r q_{\mathrm{L}}(m, r)+m \Phi_{\mathrm{L}}(m, r) \\
& +(2-m) \Phi_{\mathrm{N}}(m, r)+Q(m, r)^{2} / 2
\end{aligned}
$$

Observe that for $\mathrm{J} \in\{\mathrm{I}, \mathrm{L}, \mathrm{N}\}, \Phi_{\mathrm{J}}(m, r)=\left[p(m, r)-c_{\mathrm{J}}(r)\right] q_{\mathrm{J}}(m, r)$, where $c_{\mathrm{I}}(r)$ $=c-\varepsilon, c_{\mathrm{L}}(r)=c-\varepsilon+r$ and $c_{\mathrm{N}}(r)=c$. Since $Q(m, r)=q_{\mathrm{I}}(m, r)+m q_{\mathrm{L}}(m, r)+$ $(2-m) q_{\mathrm{N}}(m, r)$, from (9) we have

$$
W(m, r)=[p(m, r)-(c-\varepsilon)] Q(m, r)-(2-m) \varepsilon q_{\mathrm{N}}(m, r)+Q(m, r)^{2} / 2
$$

Since $p(m, r)=a-Q(m, r)$, we then have

$$
W(m, r)=[2(a-c+\varepsilon)-Q(m, r)] Q(m, r) / 2-(2-m) \varepsilon q_{\mathrm{N}}(m, r)
$$

For $i=1,2$, let us denote the equilibrium royalty rate under Regime $i$ by $r_{i}$. From Propositions 1 and 2, it follows that when the licensing policy involves a subsidy under Regime 1 (i.e. $r_{1}<0$ ), we have $m=1$. Then from (10), the welfare under Regime 1 is given by

$$
W_{1}=\left[2(a-c+\varepsilon)-Q\left(1, r_{1}\right)\right] Q\left(1, r_{1}\right) / 2-\varepsilon q_{\mathrm{N}}\left(1, r_{1}\right)
$$

\footnotetext{
${ }^{10} \mathrm{As}$ before, we use the same notation for both outsider and incumbent innovators.

(C) Blackwell Publishing Ltd and The University of Manchester, 2005.
} 
Under Regime 2, we have $m=2$, so the welfare is given by

$$
W_{2}=\left[2(a-c+\varepsilon)-Q\left(2, r_{2}\right)\right] Q\left(2, r_{2}\right) / 2
$$

Note that $[2(a-c+\varepsilon)-Q] Q$ is increasing in $Q$ for $0<Q<a-c+\varepsilon$. Now observe that under both regimes the industry output is always less than $a-$ $c+\varepsilon$, which is the competitive output with the reduced cost. Thus, $W_{2}$ and the first term of $W_{1}$ are both increasing in industry output. The second term of $W_{1}, \varepsilon q_{\mathrm{N}}\left(1, r_{1}\right)$, measures the cost of exclusion: this is the additional cost that the non-licensee firm has to pay for not having the innovation. Then from (11) and (12), it follows that $W_{1}$ would be higher than $W_{2}$ if the industry output under Regime 1 were sufficiently higher than the output under Regime 2 so as to outweigh the cost of exclusion. Recall from Proposition 1 that in the case of an outsider innovator, when $\varepsilon \geq(a-c) / 2$, Regime 1 results in a monopoly with price $c$. Since the non-licensee firm does not produce, the cost of exclusion is obviously zero. So, the regime that results in higher industry output will have a higher welfare for this case. For sufficiently significant innovations (specifically, $\varepsilon>2(a-c) / 3$ ), the duopoly price under Regime 2 falls below $c$ and, as a consequence, welfare is higher under this regime, as stated in part 3 of Proposition 3.

Let us now briefly discuss the role of antitrust policies in this regard (see Gilbert and Shapiro (1997) for a detailed discussion of antitrust issues in regard to licensing). Our analysis shows that subsidy-based licensing necessarily involves exclusive licensing. This can raise antitrust concerns, especially in the case of an incumbent innovator:

[A]ntitrust analysis of intellectual property licensing arrangements examines
whether the relationship among the parties to the arrangement is pri-
marily horizontal or vertical in nature, or whether it has substantial aspects of
both. A licensing arrangement has a vertical component when it affects activ-
ities that are in a complementary relationship, as is typically the case in a licens-
ing arrangement... For analytical purposes, the Agencies ordinarily will treat
a relationship between a licensor and its licensees [.. . . as horizontal when they
would have been actual or likely potential competitors in a relevant market in
the absence of the license... Generally, an exclusive license may raise antitrust
concerns only if the licensees themselves, or the licensor and its licensees, are
in a horizontal relationship. ${ }^{11}$

As we have seen, Regime 2 eliminates exclusive licensing, so that diffusion of the innovation is higher under this regime. However, we have also shown that the social welfare could be lower under this regime. Thus, diffusion and welfare might not always work in the same direction and exclusive licensing might not necessarily be detrimental to welfare. Our analysis suggests that factors like the nature of the market and significance of the innovation play important roles in this regard.

\footnotetext{
${ }^{11}$ Antitrust Guidelines for the Licensing of Intellectual Property, issued by the US Department of Justice and the Federal Trade Commission, 1995.
}

(C) Blackwell Publishing Ltd and The University of Manchester, 2005. 
To conclude, we observe that although subsidy-based licensing could be theoretically optimal, subsidy is rarely, if ever, observed in actual licensing practices. There are two plausible explanations behind this. First, licensing based on subsidy necessarily involves exclusive licensing. While subsidy itself is not expressly prohibited by law, we have already seen that exclusive licensing might be frowned upon by antitrust authorities. Such indirect legal barriers could prevent an innovator from charging a subsidy. Second, a per-unit subsidy might be present implicitly in the licensing contract. The main effect of a per-unit subsidy is to make a licensee firm more aggressive by shifting its best response function. In many cases, a licensing contract might have provisions for sales promotion or job training in new technology adoption. ${ }^{12}$ In fact, empirical studies suggest that licensing contracts often involve transmission of knowhow and technical assistance to the licensee (e.g. Caves et al., 1983; Macho-Stadler et al., 1996). If these factors lead to an effective reduction in cost for every unit of output, they essentially play the role of a per-unit subsidy.

\section{APPENDIX}

Notations: We denote by $q$ and $\Phi$, with suitable subscripts, the individual Cournot output and profit respectively. $q_{\mathrm{L}}(m, r)$ and $q_{\mathrm{N}}(m, r)$ denote the Cournot output of a licensee and a non-licensee firm respectively when there are $m$ licensees and the rate of royalty is $r$. Similarly, $\Phi_{\mathrm{L}}(m, r)$ and $\Phi_{\mathrm{N}}(m, r)$ denote the respective Cournot profits. When the innovator is an incumbent firm, $q_{\mathrm{I}}(m, r)$ and $\Phi_{\mathrm{I}}(m, r)$ denote respectively the Cournot output and profit of the innovator. The payoff of the innovator, industry output and social welfare are denoted by $\Pi(m, r), Q(m, r)$ and $W(m, r)$ respectively. The modified AR policy $\langle 2, r, f(2, r)\rangle$, where the minimum bid $f(2, r)$ is given by (4), will be simply denoted by $\langle 2, r\rangle$. We also denote $x \equiv(a-c) / \varepsilon$.

For both outsider and incumbent innovators, we state certain intermediary lemmas that will be used to prove the main results. The proofs are straightforward and hence omitted.

\section{An Outsider Innovator}

Lemma O1: Suppose $0<\varepsilon<\min (a-c, c)$ and let

$$
\beta_{1}=\varepsilon-(a-c) \quad \beta_{2}=\varepsilon-(a-c) / 2 \quad \beta_{3}=(a-c) / 2+\varepsilon \quad \beta_{4}=a-c+\varepsilon
$$

Then $\beta_{1}<\beta_{2}<\varepsilon<\beta_{3}<\beta_{4}$.

Lemma O2: When there is no licensee, $q_{\mathrm{N}}(0, r)=(a-c) / 3$ and $\Phi_{\mathrm{N}}(0, r)=$ $(a-c)^{2} / 9$.

\footnotetext{
${ }^{12} \mathrm{We}$ are grateful to an anonymous referee for suggesting this explanation.

C Blackwell Publishing Ltd and The University of Manchester, 2005.
} 
Lemma O3: The following hold when there is only one licensee.

1. $r \leq \beta_{1}: q_{\mathrm{L}}(1, r)=(a-c+\varepsilon-r) / 2, q_{\mathrm{N}}(1, r)=0$.

2. $r \in\left[\beta_{1}, \beta_{3}\right]: q_{\mathrm{L}}(1, r)=[a-c+2(\varepsilon-r)] / 3, q_{\mathrm{N}}(1, r)=(a-c-\varepsilon+r) / 3$.

3. $r \geq \beta_{3}: q_{\mathrm{L}}(1, r)=0, q_{\mathrm{N}}(1, r)=(a-c) / 2$.

For all cases, $\Phi_{\mathrm{J}}(1, r)=\left[q_{\mathrm{J}}(1, r)\right]^{2}$ for $\mathbf{J} \in\{\mathrm{L}, \mathbf{N}\}$.

Lemma 04: When both firms are licensees, $q_{\mathrm{L}}(2, r)=(a-c+\varepsilon-r) / 3$ if $r \leq \beta_{4}$ and $q_{\mathrm{L}}(2, r)=0$ otherwise. Moreover, $\Phi_{\mathrm{L}}(2, r)=\left[q_{\mathrm{L}}(2, r)\right]^{2}$.

Lemma 05: When there is only one licensee and $r \geq \beta_{3}$, the innovator's payoff from any FR or AR policy where the rate of royalty is $r$ is at most zero.

\section{An Incumbent Innovator}

Lemma I1: Let $0<\varepsilon<\min (a-c, c)$. For $m \in\{1,2\}$, let

$$
\begin{aligned}
& \theta_{1}(m)=(c-a-\varepsilon) / m \quad \theta_{2}(m)=[c-a+(m+1) \varepsilon] / m \\
& \theta_{3}(m)=[a-c+(3-m) \varepsilon] /(4-m)
\end{aligned}
$$

Then $\theta_{2}(1)<\theta_{2}(2)$ and $\theta_{1}(2)<\theta_{2}(2)<\theta_{3}(1)<\theta_{3}(2)$. Further, for $m \in\{1,2\}, \theta_{1}(m)<0$ $<\theta_{3}(m)$.

Lemma I2: The following hold when there is only one licensee.

1. $r \leq \theta_{1}(1): q_{\mathrm{I}}(1, r)=0, q_{\mathrm{L}}(1, r)=(a-c+\varepsilon-r) / 2, q_{\mathrm{N}}(1, r)=0$.

2. $r \in\left[\theta_{1}(1), \theta_{2}(1)\right]: q_{\mathrm{I}}(1, r)=(a-c+\varepsilon+r) / 3, q_{\mathrm{L}}(1, r)=(a-c+\varepsilon-2 r) / 3, q_{\mathrm{N}}(1, r)=$ 0 .

3. $r \in\left[\theta_{2}(1), \theta_{3}(1)\right]: q_{\mathrm{I}}(1, r)=(a-c+2 \varepsilon+r) / 4, q_{\mathrm{L}}(1, r)=(a-c+2 \varepsilon-3 r) / 4, q_{\mathrm{N}}(1, r)$ $=(a-c-2 \varepsilon+r) / 4$.

4. $r \geq \theta_{3}(1): q_{\mathrm{I}}(1, r)=(a-c+2 \varepsilon) / 3, q_{\mathrm{L}}(1, r)=0, q_{\mathrm{N}}(1, r)=(a-c-\varepsilon) / 3$.

In all cases, $\Phi_{\mathrm{J}}(1, r)=\left[q_{\mathrm{J}}(1, r)\right]^{2}$ for $\mathrm{J} \in\{\mathrm{I}, \mathrm{L}, \mathrm{N}\}$.

Lemma I3: The following hold when both firms are licensees.

1. $r \leq \theta_{1}(2): q_{\mathrm{I}}(2, r)=0, q_{\mathrm{L}}(2, r)=(a-c+\varepsilon-r) / 3$.

2. $r \in\left[\theta_{\mathrm{l}}(2), \theta_{3}(2)\right]: q_{\mathrm{I}}(2, r)=(a-c+\varepsilon+2 r) / 4, q_{\mathrm{L}}(2, r)=(a-c+\varepsilon-2 r) / 4$.

3. $r \geq \theta_{3}(2): q_{\mathrm{I}}(2, r)=(a-c+\varepsilon) / 2, q_{\mathrm{L}}(2, r)=0$.

In all cases, $\Phi_{\mathrm{J}}(2, r)=\left[q_{\mathrm{J}}(2, r)\right]^{2}$ for $\mathrm{J} \in\{\mathrm{I}, \mathrm{L}\}$.

Lemma I4: Suppose either $r \leq \max \left[\theta_{1}(2), \theta_{2}(1)\right]$ or $r \geq \theta_{3}(1)$. Then $\Pi(2, r) \leq$ $\Pi(2, \varepsilon)$. 


\section{Proof of Lemma 1}

An Outsider Innovator. When $r \leq \beta_{1}, \Pi_{\mathrm{FR}}(1, r)$ is maximized at $r=\beta_{1}$. When $r \geq \beta_{3}$, $\Pi_{\mathrm{FR}}(1, r) \leq 0$. So it is enough to consider $r \in\left[\beta_{1}, \beta_{3}\right]$. In what follows, we show that

$$
\max _{r} \Pi_{\mathrm{FR}}(1, r)=\Pi_{\mathrm{FR}}\left(1, r^{*}\right) \leq \Pi_{\mathrm{AR}}\left(1, r^{*}\right)
$$

Note that $\Pi_{\mathrm{FR}}(1, r)-\Pi_{\mathrm{AR}}(1, r)=\Phi_{\mathrm{N}}(1, r)-\Phi_{\mathrm{N}}(0, r)$. Since $\Phi_{\mathrm{N}}(m, r)=\left[q_{\mathrm{N}}(m, r)\right]^{2}$, we have

$$
\Pi_{\mathrm{FR}}(1, r) \leq \Pi_{\mathrm{AR}}(1, r) \Leftrightarrow q_{\mathrm{N}}(1, r) \leq q_{\mathrm{N}}(0, r)
$$

From Lemmas $\mathrm{O} 2$ and $\mathrm{O} 3$, it follows that when $r \in\left[\beta_{1}, \beta_{3}\right]$

$$
q_{\mathrm{N}}(0, r)-q_{\mathrm{N}}(1, r)=(\varepsilon-r) / 3 \geq 0 \Leftrightarrow r \leq \varepsilon
$$

To prove (13), thus, it is enough to show that $r^{*} \leq \varepsilon$. We note that $\Pi_{\mathrm{FR}}(1, r)$ is a quadratic function in $r$ and it is decreasing at $r=\varepsilon$. Noting that $\varepsilon>\beta_{1}$, using standard properties of quadratic functions, we conclude that when $r \in\left[\beta_{1}, \beta_{3}\right]$ the maximum of $\Pi_{\mathrm{FR}}(1, r)$ is attained at some $r^{*} \leq \varepsilon$. This completes the proof of Lemma 1 in the case of an outsider innovator.

An Incumbent Innovator. Note that when $r \geq \theta_{3}(1), \Pi_{\mathrm{FR}}(1, r) \leq 0$. When $r \leq \theta_{1}(1)$, $\Pi_{\mathrm{FR}}(1, r)$ is maximized at $r=\theta_{1}(1)$. When $r \in\left[\theta_{1}(1), \theta_{2}(1)\right]$, the maximum is attained at $r=\theta_{2}(1)$. So it is enough to consider $r \in\left[\theta_{2}(1), \theta_{3}(1)\right]$. For this case, we show that

$$
\max _{r} \Pi_{\mathrm{FR}}(1, r)=\Pi_{\mathrm{FR}}\left(1, r^{*}\right) \leq \Pi_{\mathrm{AR}}\left(1, r^{*}\right)
$$

Note as before that $\Pi_{\mathrm{FR}}(1, r) \leq \Pi_{\mathrm{AR}}(1, r)$ iff $q_{\mathrm{N}}(1, r) \leq q_{\mathrm{N}}(0, r)$. From Lemmas I3 and I4, it follows that when $r \in\left[\theta_{2}(1), \theta_{3}(2)\right]$ then

$$
q_{\mathrm{N}}(0, r)-q_{\mathrm{N}}(1, r)=(\varepsilon-r) / 4 \geq 0 \Leftrightarrow r \leq \varepsilon
$$

To prove (14) it is then enough to show that $r^{*} \leq \varepsilon$, which follows by a similar argument as in the case of an outsider innovator by noting that $\varepsilon>\theta_{2}(1), \Pi_{\mathrm{FR}}(1, r)$ is a quadratic function in $r$ and it is decreasing at $r=\varepsilon$. This completes the proof of Lemma 1 in the case of an incumbent innovator.

Remark: The conclusion of Lemma 1 holds under both regimes.

\section{Proof of Proposition 1}

To prove Proposition 1, we determine the optimal policy by comparing the payoffs of the following two cases: (a) the license is sold to both firms and (b) the license is sold to only one firm.

(a) The License is Sold to Both Firms. The payoff of the innovator is $\Pi(2, r)=$ $2 r q_{\mathrm{L}}(2, r)+2\left[\Phi_{\mathrm{L}}(2, r)-\Phi_{\mathrm{N}}(1, r)\right]$. From Lemmas O3 and O4, it follows that $\Pi(2, r) \leq$ 0 for $r \geq \beta_{4}$. Further, $\Pi(2, r)$ is maximized at $r=\beta_{3}$ for $r \in\left[\beta_{3}, \beta_{4}\right]$ and at $r=\beta_{1}$ for $r$ $\leq \beta_{1}$. So it is enough to consider $r \in\left[\beta_{1}, \beta_{3}\right]$, in which case we have 


$$
\Pi(2, r)=2 r(a-c+\varepsilon-r) / 3+2(a-c+\varepsilon-r)^{2} / 9-2(a-c-\varepsilon+r)^{2} / 9
$$

The maximum is attained at $r=\bar{r} \in\left(\beta_{1}, \beta_{3}\right)$, where

$$
\bar{r} \equiv \varepsilon / 2-(a-c) / 6
$$

The payoff of the innovator is given by

$$
\Pi(2, \bar{r})=\left[(a-c)^{2}+42(a-c) \varepsilon+9 \varepsilon^{2}\right] / 54
$$

Conclusion 01: Under Regime 1, when the innovator sells the license to both firms, the optimal licensing policy is $\langle 2, \bar{r}\rangle$, where $\bar{r}$ is given by (15) and the payoff is given by (16).

Now consider Regime 2 when there is restriction on negative royalty. We have shown that the unrestricted maximum of $\Pi(2, r)$ is attained at $r=\bar{r}$, given by (15). Since $\bar{r} \geq 0$ iff $\varepsilon \geq(a-c) / 3$, the maximum is attained at $r=\bar{r}$ for $\varepsilon \geq(a-c) / 3$ and the payoff is given by (16). When $\varepsilon \leq(a-c) / 3$, the maximum is attained at $r=0$ and the payoff is given by

$$
\Pi(2,0)=2\left[(a-c+\varepsilon)^{2} / 9-(a-c-\varepsilon)^{2} / 9\right]=8(a-c) \varepsilon / 9
$$

Conclusion O2: Under Regime 2, when the innovator sells the license to both firms, the optimal licensing policy is $\langle 2,0\rangle$ and the payoff is given by (17) when $\varepsilon \leq(a-c) / 3$. When $\varepsilon \geq(a-c) / 3$, the policy is $\langle 2, \bar{r}\rangle$, where $\bar{r}$ is given by (15) and the payoff is given by (16).

(b) The License is Sold to Only One Firm. The payoff of the innovator is $\Pi(1$, $r)=r q_{\mathrm{L}}(1, r)+\left[\Phi_{\mathrm{L}}(1, r)-\Phi_{\mathrm{N}}(1, r)\right]$. From Lemma O3, it follows that $\Pi(1, r) \leq 0$ for $r \geq \beta_{3}$ and $\Pi(1, r)$ is maximized at $r=\beta_{1}$ for $r \leq \beta_{1}$. So it is enough to consider $r \in\left[\beta_{1}, \beta_{3}\right]$, in which case we have

$$
\Pi(1, r)=r[a-c+2(\varepsilon-r)] / 3+[a-c+2(\varepsilon-r)]^{2} / 9-(a-c-\varepsilon+r)^{2} / 9
$$

The unconstrained maximum is attained at $r=\tilde{r}$, where $\tilde{r} \equiv-(a-c) / 2<0<\beta_{3}$. Since $\tilde{r}-\beta_{1}=(a-c-2 \varepsilon) / 2$, we have

$$
\tilde{r} \leq \beta_{1} \Leftrightarrow \varepsilon \geq(a-c) / 2
$$

Regime 1: To find the optimal policy under Regime 1, we consider the following two cases.

Case $(i): \varepsilon \leq(a-c) / 2$. For this case, from (19), the maximum payoff of the innovator is attained at $r=\bar{r}$. Since $\bar{r} \in\left(\beta_{1}, \beta_{3}\right)$, from Lemma O2, we conclude that both firms continue to operate. However, the difference in the respective Cournot profits of a licensee and a non-licensee is paid to the innovator as the winning bid. 
Thus, both firms earn the same payoff, which is the Cournot profit of the non-licensee. The payoff of the innovator from the policy $\langle 1, \tilde{r}\rangle$ is given by

$$
\Pi(1, \tilde{r})=\left[(a-c)^{2}+8(a-c) \varepsilon+4 \varepsilon^{2}\right] / 12
$$

Case (ii): $\varepsilon \geq(a-c) / 2$. For this case, due to (19), the maximum is attained at $r=$ $\beta_{1}$. Replacing $r=\beta_{1}$ in (18) we find that the payoff of the innovator is $(a-c) \varepsilon$. Note from Lemma $\mathrm{O} 2$ that $q_{\mathrm{N}}\left(1, \beta_{1}\right)=0$, i.e. when there is only one licensee and $r=\beta_{1}$, then the non-licensee firm drops out of the market, so that the sole licensee becomes a monopolist. However, the licensee firm pays the entire monopoly profit as the winning bid, so that both firms earn zero payoff.

Conclusion O3: Under Regime 1, the following hold when the license is sold exclusively to a single firm.

(i) For $\varepsilon \leq(a-c) / 2$, the payoff is maximized at the policy $\langle 1, \tilde{r}\rangle$ and it is given by (20).

(ii) For $\varepsilon \geq(a-c) / 2$, the payoff of the innovator is maximized at the policy $\left\langle 1, \beta_{1}\right\rangle$ and it is $(a-c) \varepsilon$.

Regime 2: Since the unconstrained maximum is attained at $\tilde{r}=-(a-c) / 2$, the optimal rate of royalty is zero under Regime 2 and the payoff of the innovator is

$$
\Pi(1,0)=(a-c+2 \varepsilon)^{2} / 9-(a-c-\varepsilon)^{2} / 9
$$

Conclusion O4: Under Regime 2, when the license is sold to only one firm, the optimal licensing policy is $\langle 1,0\rangle$ and the payoff of the innovator is given by (21).

Proof of Part 1: We use Conclusions $\mathrm{O} 1$ and $\mathrm{O} 3$ to prove part 1.

Case 1: $\varepsilon \leq(a-c) / 2$. For this case, $\Pi(2, \bar{r}) \geq \Pi(1, \bar{r})$ iff $g(x) \leq 0$, where $g(x)=7 x^{2}$ $-12 x+18$. Since $g(x)>0$ for all $x$, the optimal licensing policy for this case is $\langle 1, \tilde{r}\rangle$, where $\bar{r}=-(a-c) / 2$. For this case, both firms continue to operate.

Case 2: $\varepsilon \geq(a-c) / 2$. Observe that $\Pi(2, \bar{r}) \geq(a-c) \varepsilon$ iff $f(x) \geq 0$, where $f(x)=x^{2}-$ $12 x+9$. Since $a-c>\varepsilon$ and $\varepsilon \geq(a-c) / 2$, we have $1<x \leq 2$. Noting that $f(x)<0$ for all $x \in[1,2]$, we conclude that for $\varepsilon \geq(a-c) / 2$ the optimal licensing policy for the innovator is to sell the license exclusively to only one firm using the policy $\left\langle 1, \beta_{1}\right\rangle$, where $\beta_{1}=\varepsilon-(a-c)$ and the payoff of the innovator is $(a-c) \varepsilon$. For this case, the non-licensee firm drops out of the market. Then, part 1 of Proposition 1 follows from Cases 1 and 2.

Proof of Part 2: We use Conclusions $\mathrm{O} 2$ and $\mathrm{O} 4$ to prove part 2. For $\varepsilon \geq(a-c) / 3$, we compare $\Pi(2, \bar{r})$ and $\Pi(1,0)$. From (16) and (21), it follows that $\Pi(2, \bar{r})>$ 
$\Pi(1,0)$ for all $\varepsilon<a-c$. For $\varepsilon \leq(a-c) / 3$, we compare $\Pi(2,0)$ and $\Pi(1,0)$. From $(17)$ and $(21)$, we have $\Pi(2,0)-\Pi(1,0)=[2(a-c)-3 \varepsilon] \varepsilon / 9>0$ for $\varepsilon \leq(a-c) / 3$. This completes the proof of part 2 of Proposition 1.

\section{Proof of Proposition 2}

We prove Proposition 2 by considering the following possible cases.

(a) The License is Sold to Both Firms. The payoff of the innovator is $\Pi(2, r)=$ $\Phi_{\mathrm{I}}(2, r)+2 r q_{\mathrm{L}}(2, r)+2\left[\Phi_{\mathrm{L}}(2, r)-\Phi_{\mathrm{N}}(1, r)\right]$. For $r \in\left[\max \left\{\theta_{1}(2), \theta_{2}(1)\right\}, \theta_{3}(1)\right]$, from Lemmas I 2 and I3 we have

$$
\begin{aligned}
\Pi(2, r)= & (a-c+\varepsilon+2 r)^{2} / 16+r(a-c+\varepsilon-2 r) / 2+(a-c+\varepsilon-2 r)^{2} / 8 \\
& -(a-c-2 \varepsilon+r)^{2} / 8
\end{aligned}
$$

The maximum of $\Pi(2, r)$ is attained at $r=\varepsilon$ and the payoff is given by

$$
\Pi(2, \varepsilon)=\left[(a-c)^{2}+14(a-c) \varepsilon+\varepsilon^{2}\right] / 16
$$

By Lemma I4, it follows that this maximum is also the global maximum. Since the unconstrained optimal policy does not involve any negative royalty, this is the optimal policy for both regimes when the license is sold to both firms.

Conclusion I1: Under both regimes, when both firms are licensees, the payoff of the innovator is maximized at the royalty policy with rate of royalty $\varepsilon$ and it is given by (22).

(b) The License is Sold to Only One Firm. The payoff is $\Pi(1, r)=\Phi_{\mathrm{I}}(1, r)+$ $r q_{\mathrm{L}}(1, r)+\left[\Phi_{\mathrm{L}}(1, r)-\Phi_{\mathrm{N}}(1, r)\right]$. Due to Lemma I4, we restrict $r \in\left[\theta_{2}(1), \theta_{3}(1)\right]$ in which case, from Lemmas I 2 and I3, it follows that

$$
\begin{aligned}
\Pi(1, r)= & (a-c+2 \varepsilon+r)^{2} / 16+r(a-c+2 \varepsilon-3 r) / 4+(a-c+2 \varepsilon-3 r)^{2} / 16 \\
& -(a-c-2 \varepsilon+r)^{2} / 16
\end{aligned}
$$

The unrestricted maximum is attained at $r_{\mathrm{A}}$, where

$$
r_{\mathrm{A}} \equiv[2 \varepsilon-(a-c)] / 3
$$

Regime 1: Note that $r_{\mathrm{A}}<\theta_{3}(1)$. Since $r_{\mathrm{A}}-\theta_{2}(1)=2(a-c-2 \varepsilon) / 3$, we have

$$
r_{\mathrm{A}} \geq \theta_{2}(1) \Leftrightarrow \varepsilon \leq(a-c) / 2
$$

Then, from (23) and (24) we conclude that under Regime $1 \Pi(1, r)$ is maximized at $r$ $=r_{\mathrm{A}}$ when $\varepsilon \leq(a-c) / 2$. When $\varepsilon \geq(a-c) / 2$, it is maximized at $r=\theta_{2}(1)$. The next conclusion follows by noting that $\Pi\left(1, \theta_{2}(1)\right)=(a-c) \varepsilon$ and

$$
\Pi\left(1, r_{\mathrm{A}}\right)=\left[(a-c)^{2}+8(a-c) \varepsilon+4 \varepsilon^{2}\right] / 12
$$


Conclusion I2: Under Regime 1, when an incumbent innovator sells the license to only one firm, the following hold.

(i) For $\varepsilon \leq(a-c) / 2$, the payoff of the innovator is maximized at the policy $\left\langle 1, r_{\mathrm{A}}\right\rangle$ where $r_{\mathrm{A}}$ is given by (23) and the payoff is given by (25).

(ii) For $\varepsilon \geq(a-c) / 2$, the payoff is maximized at the policy $\left\langle 1, \theta_{2}(1)\right\rangle$ and is $(a-c) \varepsilon$.

Regime 2: Recall that $\theta_{2}(1)=2 \varepsilon-(a-c)$, so that $\theta_{1} \geq 0$ when $\varepsilon \geq(a-c) / 2$, in which case the payoff is maximized at $r=\theta_{2}(1)$ and is given by $(a-c) \varepsilon$. When $\varepsilon \leq(a-c) / 2$, from (23), it follows that $r_{\mathrm{A}} \leq 0$, so that the maximum under Regime 2 is attained at $r=0$ and the payoff is

$$
\Pi(1,0)=(a-c+2 \varepsilon)^{2} / 16+(a-c+2 \varepsilon)^{2} / 16-(a-c-2 \varepsilon)^{2} / 16
$$

Conclusion 13: Under Regime 2, the following hold when an incumbent innovator sells the license to only one firm.

(i) For $\varepsilon \leq(a-c) / 2$, the payoff of the innovator is maximized at the policy $\langle 1,0\rangle$ and it is given by (26).

(ii) For $\varepsilon \geq(a-c) / 2$, the payoff is maximized at the policy $\left\langle 1, \theta_{2}(1)\right\rangle$ and it is given by $(a-c) \varepsilon$.

Proof of Part 1: To prove part 1 of Proposition 2, we use Conclusions I1 and I2. From (22), it follows that $\Pi(2, \varepsilon)-(a-c) \varepsilon=(a-c-\varepsilon)^{2} / 16>0$, so that when $\varepsilon \geq(a$ $-c) / 2$ the optimal policy is the royalty policy $\langle 2, \varepsilon, 0\rangle$. When $\varepsilon \leq(a-c) / 2$, we compare $\Pi(2, \varepsilon)$ and $\Pi\left(1, r_{\mathrm{A}}\right)$. From (22) and (25), we have $\Pi(2, \varepsilon) \geq \Pi\left(1, r_{\mathrm{A}}\right)$ iff $h(x) \leq 0, h(x)$ $=12\left(x^{2}-10 x+13\right)$. We are considering $x>2$ and $h(x)$ has only one root above $2: k$ $\equiv 5+\sqrt{ } 108 / 3$. Further, $h(x)<0$ for $2<x<k$ and $h(x)>0$ for $x>k$. Thus, the optimal policy is $\langle 2, \varepsilon, 0\rangle$ for $\varepsilon \geq(a-c) / k$, and it is the policy $\left\langle 1, r_{\mathrm{A}}\right\rangle$ otherwise, where $r_{\mathrm{A}}$ is given by (23). This completes the proof of part 1 of Proposition 2.

Proof of Part 2: To prove part 2, we use Conclusions I1 and I3. We have already shown that $\Pi(2, \varepsilon)>(a-c) \varepsilon$, so that for $\varepsilon \geq(a-c) / 2$ the optimal policy is $\langle 2, \varepsilon, 0\rangle$. When $\varepsilon \leq(a-c) / 2$, we compare $\Pi(2, \varepsilon)$ and $\Pi(1,0)$. Noting that, for $\varepsilon \leq(a-c) / 2$, $\Pi(2, \varepsilon)-\Pi(1,0)=\varepsilon[2(a-c)-3 \varepsilon] / 16>0$, we conclude that optimal policy in this region is also $\langle 2, \varepsilon, 0\rangle$. This completes the proof of Proposition 2.

\section{Proof of Proposition 3}

Part 1 . We have already proved part 1 in the main text.

Part 2. For part 2, let us first consider an outsider innovator. When $\varepsilon \leq(a-c) / 3$, the equilibrium royalty rate under Regime 1 is $\tilde{r} \equiv-(a-c) / 2$ and the industry output is $Q(1, \tilde{r})=[5(a-c)+2 \varepsilon] / 6$, while under Regime 2 the royalty is zero and the output is $Q(2,0)=2(a-c+\varepsilon) / 3$. Observing that $Q(1, \tilde{r})>Q(2,0)$ for $\varepsilon \leq(a-c) / 3$, it follows 
that the consumers are better off under Regime 1 for this case. When $(a-c) / 3 \leq \varepsilon \leq$ $(a-c) / 2$, the industry output under Regime 1 is still $Q(1, \tilde{r})$, while under Regime 2 the royalty is $\bar{r} \equiv \varepsilon / 2-(a-c) / 6$ and the output is $Q(2, \tilde{r})=[7(a-c)+3 \varepsilon] / 9$. Observing that $Q(1, \tilde{r})>Q(2, \bar{r})$, it follows that the consumers are better off under Regime 1 for this case. Finally, consider $\varepsilon \geq(a-c) / 2$. Then the Cournot price under Regime 1 is $c$, so the industry output is $a-c$. Under Regime 2, the output for this case is still $Q(2, \bar{r})$. Observing that $a-c \geq Q(2, \bar{r})$ if $\varepsilon \leq 2(a-c) / 3$, the result follows for an outsider innovator. Next consider an incumbent innovator. From Proposition 2, it follows that when $\varepsilon \geq(a-c) / k$ both regimes result in the same outcome. For $\varepsilon \leq(a-c) / k$, the equilibrium royalty rate under Regime 1 is $r_{\mathrm{A}} \equiv[2 \varepsilon-(a-c)] / 3$ and the industry output is $Q\left(1, r_{\mathrm{A}}\right)=[13(a-c)+6 \varepsilon] / 16$. Under Regime 2 , the royalty is $\varepsilon$ and the output is $Q(2, \varepsilon)=[3(a-c)+\varepsilon] / 4$. The result follows by observing that $Q\left(1, r_{\mathrm{A}}\right)>Q(2, \varepsilon)$.

Part 3. For $i=1,2$, let us denote by $r_{i}$ and $W_{i}$ the equilibrium royalty rate and social welfare respectively under Regime $i$. Then we have

$$
\begin{aligned}
& W_{1}=\left[2(a-c+\varepsilon)-Q\left(1, r_{1}\right)\right] Q\left(1, r_{1}\right) / 2-\varepsilon q_{\mathrm{N}}\left(1, r_{1}\right) \\
& W_{2}=\left[2(a-c+\varepsilon)-Q\left(2, r_{2}\right)\right] Q\left(2, r_{2}\right) / 2
\end{aligned}
$$

For $\varepsilon \leq(a-c) / 2$, we have $W_{1}=\left[35(a-c)^{2}+52(a-c) \varepsilon+44 \varepsilon^{2}\right] / 72$. For $\varepsilon \leq(a-c) / 3$, we have $W_{2}=4(a-c+\varepsilon)^{2} / 9$ and $W_{1}>W_{2}$. For $(a-c) / 3 \leq \varepsilon \leq(a-c) / 2, W_{2}=[11(a-c)$ $+15 \varepsilon][7(a-c)+3 \varepsilon] / 162$ and standard comparisons show that $W_{1}>W_{2}$ for this case. Finally, consider $\varepsilon \geq(a-c) / 2$, in which case $q_{\mathrm{N}}\left(1, r_{1}\right)=0$. Note that the industry output under both regimes is less than $a-c+\varepsilon$, which is the competitive output with the innovation. Since $[2(a-c+\varepsilon)-Q] Q / 2$ is increasing in $Q$ for $Q<a-c+\varepsilon$, the result follows from the conclusion of part 2 regarding an outsider innovator.

Part 4. From Proposition 2, it follows that $W_{1}=W_{2}$ for $\varepsilon \geq(a-c) / k$. For $\varepsilon<(a-$ $c) / k$, the optimal policy under Regime 1 is $\left\langle 1, r_{\mathrm{A}}\right\rangle$, where $r_{\mathrm{A}}=[2 \varepsilon-(a-c)] / 3$ and the welfare is given by $W_{1}=\left[35(a-c)^{2}+52(a-c) / \varepsilon+44 \varepsilon^{2}\right] / 72$. Under Regime 2 , the optimal policy is $\langle 2, \varepsilon, 0\rangle$ and the welfare is given by $W_{2}=[5(a-c)+7 \varepsilon][3(a-c)+$ $\varepsilon] / 32$. Comparing $W_{1}$ and $W_{2}$, it follows that $W_{1}>W_{2}$ for all $\varepsilon<a-c$. This completes the proof of the proposition.

\section{REFERENCES}

Arrow, K. J. (1962). 'Economic Welfare and the Allocation of Resources for Invention', in R. R. Nelson (ed.), The Rate and Direction of Inventive Activity: Economic and Social Factors, Princeton, NJ, Princeton University Press.

Bagwell, K. and Staiger, R. W. (1992). 'The Sensitivity of Strategic and Corrective R and D Policy in Battles for Monopoly', International Economic Review, Vol. 33, pp. 795-816.

Bulow, J. I., Geanakoplos, J. D. and Klemperer, P. D. (1985). 'Multimarket Oligopoly: Strategic Substitutes and Complements', Journal of Political Economy, Vol. 93, pp. 488-511. 
Caves, R. E., Crookell, H. and Killing, J. P. (1983). 'The Imperfect Market for Technology Licenses', Oxford Bulletin of Economics and Statistics, Vol. 45, pp. 249-267.

Fershtman, C. and Judd, K. L. (1987). 'Equilibrium Incentives in Oligopoly', American Economic Review, Vol. 77, pp. 927-940.

Gilbert R. and Shapiro, C. (1997). 'Antitrust Issues in the Licensing of Intellectual Property: The Nine No-No's Meet the Nineties', Brookings Papers on Economic Activity: Microeconomics, Vol. 1997, pp. 283-349.

Hart, O. and Holmstrom, B. (1987). 'The Theory of Contracts', in T. Bewley (ed.), Advances in Economic Theory, Fifth World Congress, Cambridge, Cambridge University Press.

Kamien, M. I. and Tauman, Y. (1984). 'The Private Value of a Patent: A Game Theoretic Analysis', Zeitschrift für Nationalökonomie, Supplement 4, pp. 93-118.

Kamien, M. I. and Tauman, Y. (1986). 'Fees Versus Royalties and the Private Value of a Patent', Quarterly Journal of Economics, Vol. 101, pp. 471-491.

Kamien, M. I. and Tauman, Y. (2002). 'Patent Licensing: The Inside Story', The Manchester School, Vol. 70, pp. 7-15.

Kamien, M. I., Oren, S. S. and Tauman, Y. (1992). 'Optimal Licensing of Costreducing Innovation', Journal of Mathematical Economics, Vol. 21, pp. 483-508.

Katz, M. L. and Shapiro, C. (1985). 'On the Licensing of Innovation', RAND Journal of Economics, Vol. 16, pp. 504-520.

Katz, M. L. and Shapiro, C. (1986). 'How to License Intangible Property', Quarterly Journal of Economics, Vol. 101, pp. 567-589.

Leahy, D. and Neary, J. P. (1997). 'Public Policy Towards R\&D in Oligopolistic Industries', American Economic Review, Vol. 87, No. 4, pp. 642-662.

Macho-Stadler, I., Martinez-Giralt, X. and Pérez-Castrillo, J. D. (1996). 'The Role of Information in Licensing Contract Design', Research Policy, Vol. 25, pp. 25-41.

Mirrlees, J. (1974). 'Notes on Welfare Economics, Information and Uncertainty', in M. Balch, D. McFadden and S. Wu (eds), Essays in Economic Behavior in Uncertainty, Amsterdam, North-Holland.

Mukherjee, A. (2002). 'Subsidy and Entry: The Role of Licensing', Oxford Economic Papers, Vol. 54, pp. 160-171.

Muniagurria, M. E. and Singh, N. (1997). 'Foreign Technology, Spillovers, and R\&D Policy', International Economic Review, Vol. 38, pp. 405-430.

Ross, S. (1973). 'The Economic Theory of Agency: The Principal's Problem', American Economic Review, Vol. 63, pp. 134-139.

Sen, D. (2002). 'Monopoly Profit in a Cournot Oligopoly', Economics Bulletin, Vol. 4, pp. 1-6.

Sen, D. (forthcoming). 'Fee Versus Royalty Reconsidered', Games and Economic Behavior, in press.

Sen, D. and Tauman, Y. (2003). 'General Licensing Schemes for a Cost-reducing Innovation', Working Paper, SUNY at Stony Brook.

Shapiro, C. (1985). 'Patent Licensing and R\&D Rivalry', American Economic Review, Papers and Proceedings, Vol. 75, pp. 25-30.

Spencer, B. J. and Brander, J. A. (1983). 'International R\&D Rivalry and Industrial Strategy', Review of Economic Studies, Vol. 50, pp. 707-722.

Wang, X. H. (1998). 'Fee Versus Royalty Licensing in a Cournot Duopoly Model', Economics Letters, Vol. 60, pp. 55-62.

Wilson, R. (1968). 'The Structure of Incentives for Decentralization under Uncertainty', in M. Guilbaud (ed.), La Decision, Paris, CNRS. 